\title{
Comparison of Maternal Serum Soluble Fms-Like Tyrosine Kinase-1/Placental Growth Factor Ratio in Preeclamptic and Normotensive Pregnant Women
}

\author{
(D) Tuğba Kılık, (D) Veli Mihmanlı, (D) Sehtap Nazlı Kılıç Çetin, (D) Orhan Şahin, (D) Gökhan Demirayak, (D) Berk Bulut \\ University of Health Sciences, İstanbul Okmeydanı Training and Research Hospital, Department of Obstetrics and Gynecology, istanbul, Turkey
}

\section{Abstract}

Objective: It has been suggested that the underlying pathology in preeclampsia is inadequate trophoblastic invasion, abnormal placentation and extensive endothelial damage. The aim of this study was to compare soluble Fms-like tyrosine kinase-1 (sFlt-1) and placental growth factor (PGF) levels and sFlt-1/PGF ratio between preeclamptic and normotensive pregnant women, and to determine the increase in sFlt-1, which is an important antiangiogenic factor in the development of preeclampsia.

Methods: Following local ethics committee approval, this prospective study was carried out with 43 pregnant women, including 20 preeclamptic and 23 normotensive pregnancies, who were admitted to our clinic. The two groups were compared in terms of gestational characteristics, sFlt-1 levels, PGF levels and sFIt-1/PGF ratio.

Results: Serum sFlt-1 levels and sFlt-1/PGF ratio were higher and serum PGF levels were lower in preeclamptic women compared to the control group. Conclusion: The sFlt-1/PGF ratio was significantly higher in preeclamptic pregnant women than in normotensive pregnant women. This higher ratio suggests that sFlt-1 has a role in the development of hypoxia and inadequate angiogenesis in preeclamptic pregnancies.

Keywords: Preeclampsia, sFlt, placental growth factor

\section{INTRODUCTION}

Preeclampsia is a multisystemic disorder characterized by hypertension accompanied by proteinuria and/or end organ damage in a normotensive woman after 20 weeks of gestation. It is seen in approximately $3-8 \%$ of pregnancies and is responsible for $18 \%$ of maternal mortality and $40 \%$ of fetal mortality worldwide $(1,2)$.

Despite its high mortality and morbidity, its etiology and pathophysiology are still unclear. Abnormal placentation with insufficient trophoblastic invasion and extensive endothelial damage is the main proposed pathology. Immunological intolerance, genetic abnormalities, metabolic and nutritional factors between fetoplacental tissue and maternal tissue are factors suggested in the pathogenesis (3).
The imbalance between angiogenic factors such as vascular endothelial growth factor (VEGF) and placental growth factor (PGF) and antiangiogenic factors such as Soluble Fms-like Tyrosine Kinase-1 (sFlt-1) and soluble endoglin (s-Eng) has been suggested to play a role in the pathogenesis of preeclampsia by causing endothelial dysfunction $(4,5)$.

sFlt-1 is over-produced in trophoblastic tissues of pregnant women who will encounter preeclampsia. sFlt-1 is a variant of the Flt-1 receptor for PGF and VEGF. The increase in maternal serum sFlt-1 levels affects the free PGF and VEGF in the circulation and causes endothelial dysfunction. Although not yet clearly explained, it is thought that the hypoxia caused by the deterioration of placental perfusion leads to over-production of antiangiogenic proteins in the placenta (6). 
The aim of this study was to determine the sFlt-1/PGF ratio in preeclampsia, which is an important cause of maternal morbidity and mortality.

\section{METHODS}

This study was planned following approval from Clinical Research Ethics Committee of İstanbul Okmeydanı Training and Research Hospital, University of Health Sciences. Forty-three pregnant women, including 20 preeclamptic and 23 normotensive women, who were admitted to department of obstetrics and gynecology between September 2016 and April 2017 and who consented, were included in the study. The patients were between the ages of 15-44 and had singleton pregnancies.

Blood pressure in each patient was measured from the brachial artery using standard measurement technique, in sitting position and the cuff at the heart level.

Ten $\mathrm{mL}$ of blood samples were drawn from the antecubital brachial vein of the patient and control group by using Vacutainer blood collection tube. The biochemistry tube was centrifuged at $3000 \mathrm{xg}$ for 10 minutes. The obtained serum was stored at $-80^{\circ} \mathrm{C}$ to be used on the day of analysis. On the day of the analysis, the eppendorf tubes were brought to room temperature, and the frozen sera were melted. The measurement of serum human sFlt-1 and PGF were performed by ELISA in the Biochemistry Laboratory of Bezmialem Vakıf University.

\section{Statistical Analysis}

Mean, standard deviation, median, minimum, maximum, frequency and ratio values were used in descriptive statistics of the data. The distribution of variables was measured by Kolmogorov-Smirnov test. Mann-Whitney U test was used for the analysis of quantitative independent data. The chi-square test was used for the analysis of qualitative independent data and the Fischer test was used when the chi-square test conditions were not met. Effect level and cut-off value were investigated with receiver operating characteristic curve. SPSS 22.0 program was used for analysis. P values less than 0.05 were considered statistically significant.

\section{RESULTS}

Forty-three pregnant women, including 20 preeclamptic and 23 normotensive women, were included in the study. The demographic and gestational characteristics of the groups are shown in Table 1. The age, gravida and parity were higher in the preeclampsia group, and but there was no difference between the groups in terms of body mass index.
Regarding sFlt-1 and PGF levels, sFlt-1 levels and sFlt-1/PGF ratio were higher and serum PGF levels were lower in preeclamptic women compared to the control group (Figures 1, 2, 3). sFlt-1 was $146.4 \pm 61.2 \mathrm{ng} / \mathrm{mL}, \mathrm{PGF}$ was $122.3 \pm 195.9 \mathrm{pg} / \mathrm{mL}$ and sFlt/ PGF ratio was $2.4 \pm 1.8$ in the preeclampsia group, and sFlt-1 was $35.0 \pm 35.8 \mathrm{ng} / \mathrm{mL}$, PGF was $172.1 \pm 194.2 \mathrm{pg} / \mathrm{mL}$ and sFlt/ PGF ratio was found to be $0.3 \pm 0.3$ in the normotensive group (Table 2).

\section{DISCUSSION}

The imbalance between angiogenic factors such as VEGF and PGF and antiangiogenic factors such as sFlt-1 and s-Eng has been suggested to play a role in the pathogenesis of preeclampsia by causing endothelial dysfunction $(4,5)$.

Leanos-Miranda et al. (7) found that proteinuria, creatinine, urea, sFlt-1 values were high and PGF values were low in preeclamptic

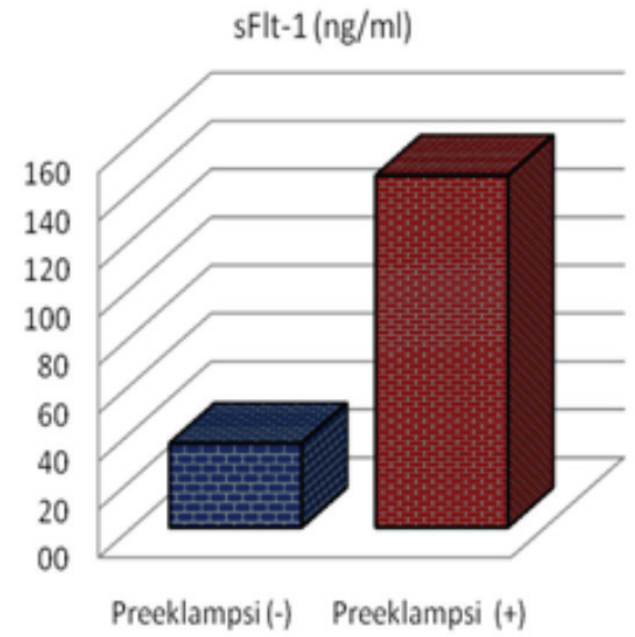

Figure 1. sFtl values $(\mathrm{ng} / \mathrm{mL})$ sFlt: Soluble Fms-like tyrosine kinase-1

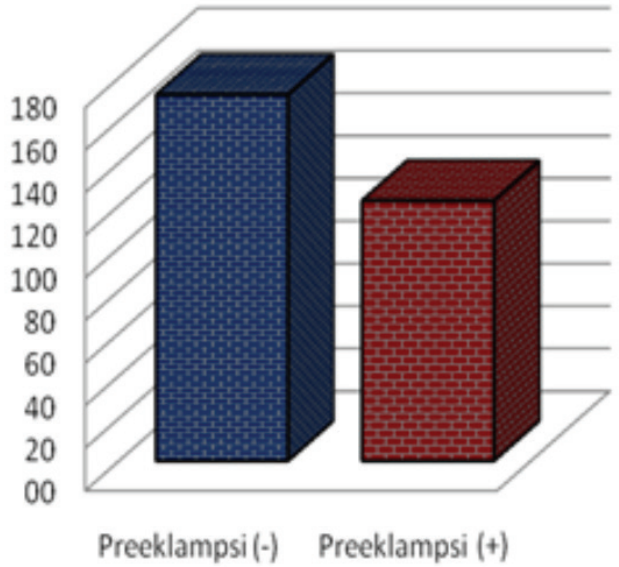

Figure 2. PGF values $(\mathrm{pg} / \mathrm{mL})$ PGF: Placental growth factor 
pregnant women. In our study, sFlt-1 levels and sFlt-1/PGF ratio were higher and serum PGF levels were lower in preeclamptic women compared to the control group.

McElrath et al. (8) found high sFlt-1 and low PGF levels in pregnant women with preeclampsia. They stated that these changes began to occur before the clinical course of the disease and were related to the severity of the disease.

Rana et al. (9) studied 616 pregnant women and found that preeclampsia developed in patients with high sFlt-1/PGF ratio and that this increase was more pronounced in pregnancies less than 34 weeks.

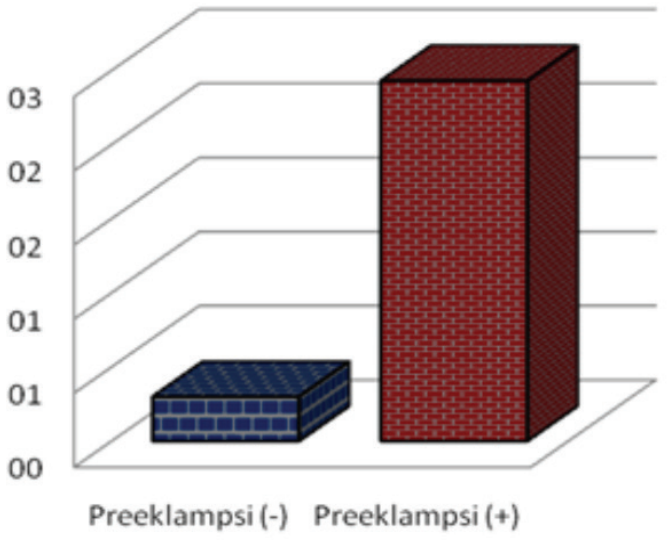

Figure 3. sFlt-1/PGF ratios

sFlt: Soluble Fms-like tyrosine kinase-1, PGF: Placental growth factor
Hunter et al. (10) only examined serum PGF level in pregnant women at risk for preeclampsia and found a negative predictive value of $98 \%$ in pregnancies less than 34 weeks and $69 \%$ in pregnancies more than 34 weeks for predicting preeclampsia in two weeks. They suggested that control of PGF levels below 34 weeks might be useful in predicting the development of preeclampsia.

Verlohren et al. (11) found the sensitivity and specificity of sFlt1 and PGF as 90\% in predicting preeclampsia in pregnancies less than 34 weeks and the sensitivity as 74\% in predicting preeclampsia in pregnancies more than 34 weeks.

Baltajian et al. (12) divided 100 women with suspected preeclampsia into two groups with high and low sFlt-1/PGF ratio, and found that negative obstetric results were more common in 43 women with a high sFlt-1/PGF ratio.

Zeisler et al. (13) stated that preeclampsia did not develop at least one week in pregnant women with low sFlt-1/PGF ratio, and that there is increased risk of developing preeclampsia in pregnant women with high ratio in four weeks and recommended increasing follow-up frequency.

\section{CONCLUSION}

In conclusion, it is important to predict pregnant women at risk for preeclampsia, and early diagnosis is necessary to reduce

\begin{tabular}{|c|c|c|c|c|c|c|c|c|c|}
\hline & & \multicolumn{3}{|c|}{ Preeclampsia (-) } & \multicolumn{3}{|c|}{ Preeclampsia $(+)$} & \multirow{2}{*}{\multicolumn{2}{|c|}{ p }} \\
\hline & & \multicolumn{2}{|c|}{ Mean \pm SD / n-\% } & Median & \multicolumn{2}{|c|}{ Mean \pm SD / n-\% } & Median & & \\
\hline \multicolumn{2}{|c|}{ BMI $\left(\mathrm{kg} / \mathrm{m}^{2}\right)$} & \multicolumn{2}{|c|}{$31.0 \pm 4.2$} & 30.5 & \multicolumn{2}{|c|}{$30.6 \pm 5.6$} & 30.3 & 0.551 & $\mathrm{~m}$ \\
\hline Smoking & No & 22 & $95.7 \%$ & & 19 & $95.0 \%$ & & 1.000 & $x^{2}$ \\
\hline \multicolumn{2}{|l|}{ Gravida } & \multicolumn{2}{|c|}{$2.4 \pm 1.6$} & 2.0 & \multicolumn{2}{|c|}{$3.5 \pm 1.8$} & 4.0 & 0.038 & $\mathrm{~m}$ \\
\hline \multicolumn{2}{|l|}{ Parity } & \multicolumn{2}{|c|}{$1.0 \pm 1.1$} & 1.0 & \multicolumn{2}{|c|}{$1.8 \pm 1.3$} & 2.0 & 0.039 & $\mathrm{~m}$ \\
\hline \multicolumn{2}{|c|}{ Systolic blood pressure (mm/Hg) } & \multicolumn{2}{|c|}{$111.7 \pm 12.7$} & 110.0 & \multicolumn{2}{|c|}{$155.0 \pm 30.3$} & 160.0 & 0.000 & $\mathrm{~m}$ \\
\hline \multicolumn{2}{|c|}{ Diastolic blood pressure $(\mathrm{mm} / \mathrm{Hg})$} & \multicolumn{2}{|c|}{$72.2 \pm 10.4$} & 70.0 & \multicolumn{2}{|c|}{$97.5 \pm 20.7$} & 100.0 & 0.000 & $\mathrm{~m}$ \\
\hline
\end{tabular}

Table 2. Comparison of sFlt-1 and PGF and sFlt/PGF ratios between groups

\begin{tabular}{|c|c|c|c|c|c|c|}
\hline & \multicolumn{2}{|l|}{ Preeclampsia (-) } & \multicolumn{2}{|l|}{ Preeclampsia (+) } & \multirow{2}{*}{\multicolumn{2}{|c|}{$p$}} \\
\hline & Mean \pm SD / n-\% & Median & Mean \pm SD / n-\% & Median & & \\
\hline sFlt-1 (ng/mL) & $35.0 \pm 35.8$ & 21.0 & $146.4 \pm 61.2$ & 136.1 & 0.000 & $\mathrm{~m}$ \\
\hline sFlt-1/PGF & $0.3 \pm 0.3$ & 0.2 & $2.4 \pm 1.8$ & 2.2 & 0.000 & $\mathrm{~m}$ \\
\hline
\end{tabular}


maternal and fetal morbidity. Although sFlt-1, PGF and sFlt/PGF ratio may be used for this purpose, large randomized controlled trials are needed for their widespread use.

\section{Ethics}

Ethics Committee Approval: This study was planned following approval from Clinical Research Ethics Committee of Okmeydanı Training and Research Hospital, University of Health Sciences (approval no: 28.06.2016/501).

Informed Consent: Written consent was obtained from the patient.

Peer-review: Externally peer-reviewed.

\section{Authorship Contributions}

Surgical and Medical Practices: T.K., S.N.K.C., Concept: T.K., V.M., Design: T.K., V.M., Data Collection or Processing: T.K., S.N.K.C.., B.B., Analysis or Interpretation: T.K., O.Ş., G.D., Literature Search: T.K., Writing: T.K., V.M.

Conflict of Interest: No conflict of interest was declared by the authors.

Financial Disclosure: The authors declared that this study received no financial support.

\section{REFERENCES}

1. Verlohren S, Galindo A, Schlembach D, Zeisler H, Herraiz I, Moertl MG, et al. An automated method for the determination of the sFIt-1/ PIGF ratio in the assesment of preeclampsia. Am J Obstet Gynecol 2010;202:161. e1-161. e11.

2. National Collaborating Centre for Women's and Children's Health, Antenatal Care: Routine Care for the Healthy Pregnant Women. Clinical Guideline. Commissioned by the National Institute for Clinical Excellence. RCOG Press: London, UK. 2008;218-27.
3. Dekker GA, Sibai BM. Pathogenesis and etiology of preeclampsia. Am J Obstet Gynecol 1998;179:1359.

4. Mutter WP, Karumanchi SA. Molecular mechanisms of preeclampsia microvascular research 2008;75:1-8.

5. Szpera Gozdziewicz A, Breborowicz GH. Endothelial dysfunction in the pathogenesis of preeclampsia. Front Biosci (Landmark Ed) 2014;19:736-46.

6. Maynard SE, Min JY, Merchan J, Lim KH, Li J, Mondal S, Libermann TA, et al. Excess placental sflt-1 may contribute to endothelial dysfunction, hypertension and proteinuria in preeclampsia. J Clin Invest 2003;111:64958.

7. Leanos-Miranda A, Campos-Galicia I, Ramirez-Valenzuela KL, ChinollaArellano ZL, Isordia-Salas I. Circulating angiogenic factors and urinary prolactin as predictors of adverse outcomes in women with preeclampsia. Hypertension 2013;61:1118-25.

8. McElrath TF, Lim K- H, Pare E, Rich-Edwards J, Pucci D, Troisi R, et al Longitudinal evaluation of predictive value for preeclampsia of circulating angiogenic factors through pregnancy. Am J Obstet Gynecol 2012;207:407. e1-7.

9. Rana S, Powe CE, Salahuddin S, Verlohren S, Perschel FH, Levine RJ, et al. Angiogenic factors and the risk of adverse outcomes in women with suspected preeclampsia. Circulation 2012;125:911-9.

10. Hunter R, Duckworth S, Seed P, Shennan A, Chappell L. PP051. Budget impact analysis of maternal plasma PIGF concentrations in women with suspected preeclampsia: the potential for improved health service usage. Pregnancy Hypertens 2013;3:85.

11. Verlohren S, Stephan H, Dechend R. Angiogenic growth factors in the diagnosis and prediction of preeclampsia. Clin Sci 2012;122:43-52.

12. Baltajian K, Bajracharya S, Salahuddin S, Berg AH, Geachchan C, Wenger JB, et al. Sequential plasma angiogenic factors levels in women with suspected preeclampsia. Am J Obstet Gynecol 2016;215:89 e1-9. e10.

13. Zeisler H, Llurba E, Chantraine F, Vatish M, Staff AC, Sennstrom M, et al. Predictive value of the sFlt-1/PIGF ratio in women with suspected preeclampsia. N Engl J Med 2016;374:13-22. 